\title{
Welcome to the Chinese Journal of Academic Radiology
}

\author{
Zhengyu Jin $^{1} \cdot$ N. Reed Dunnick ${ }^{2} \cdot$ Herbert Y. Kressel ${ }^{3} \cdot$ Maximilian Reiser $^{4}$
}

Received: 19 October 2018 / Revised: 20 November 2018 / Accepted: 20 November 2018 / Published online: 12 March 2019

(c) Springer Nature Singapore Pte Ltd. 2019

The rapid development in biomedical research in China and the increasing global reach of scientific communication have given us a strong rationale for a scientific journal of Chinese radiology. Chinese Journal of Academic Radiology as a valuable showcase provides an opportunity for the exchange of Chinese and world information for imaging research. With this journal, we believe that it has ability to enhance mutual exchange with China and other countries in the process of radiological development and promote common development.

Chinese Journal of Academic Radiology is a quarterly international journal publishing high-quality peer-reviewed research in the field of imaging basic and clinical practice. Imaging techniques will include radiography, computed tomography (CT), magnetic resonance imaging (MRI), nuclear medicine, optical imaging, ultrasound, and imageguided interventional techniques. Articles will include both clinical and translational research, showing the pathway from laboratory discovery to clinical application. We also encourage the submission of articles on data science as it pertains to radiology. Contents of this peer-reviewed journal include original articles, review (including pictorial review), editorials, short communications, case report, technical note, and letters to the editor.

To ensure its quality, many world-renown radiologists and imaging scientists from North America and Europe have enthusiastically joined our editorial board and look forward to contributing their ideas, time, and energy to this effort. Our journal strives for a rapid (up-to 3 weeks), doubleblinded peer-review process. Our international reviewers are happy to offer their sincerest constructive criticisms, comments, and suggestions for your manuscripts. Once accepted, your article will be published immediately online, where it will be available free in 2019 .

We sincerely welcome all the international academic radiologists to submit their research to the Chinese Journal of Academic Radiology https://www.springer.com/medicine/ radiology/journal/42058.

Zhengyu Jin

jin_zhengyu@163.com

1 Department of Radiology, Peking Union Medical College Hospital, Chinese Academy of Medical Sciences and Peking Union Medical College, Beijing 100730, China

2 Division of Abdominal Radiology, Michigan University Hospital, Ann Arbor, MI 48109, USA

3 Department of Radiology, Beth Israel Deaconess Medical Center, Boston, MA 02215, USA

4 Department of Radiology, Munich University Hospital, Munich 81377, Germany 